\title{
Diet and weight management by people with nonmetastatic colorectal cancer during chemotherapy: mixed methods research
}

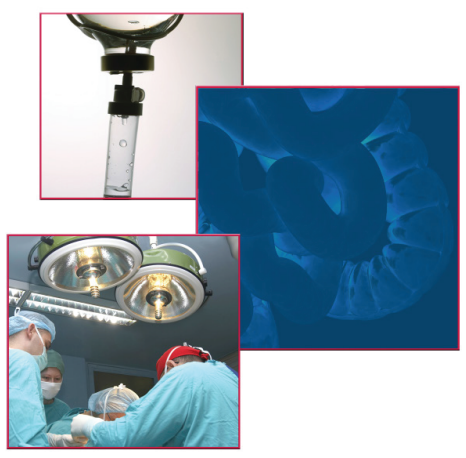

\author{
Jane B Hopkinson*,1, Catherine Kazmi ${ }^{1}$, Jayne Elias², Sally Wheelwright ${ }^{3}$, Rhiannon \\ Williams ${ }^{2}$, Ann Russell ${ }^{4} \&$ Clare Shaw ${ }^{5}$ \\ ${ }^{1}$ Cardiff University, School of Healthcare Sciences, Cardiff, Wales CF24 OAB, UK \\ ${ }^{2}$ Velindre University NHS Trust, Cardiff, Wales CF15 7QZ, UK \\ ${ }^{3}$ Macmillan Survivorship Research Group, University of Southampton, England SO17 1BJ, UK \\ ${ }^{4}$ National Institute for Health Research (NIHR), Cancer \& Nutrition Collaborative, Level E, Lab \& Path Block (MP113), Southampton \\ General Hospital, Tremona Road, Southampton SO16 6YD, UK \\ ${ }^{5}$ National Institute for Health Research (NIHR), Biomedical Research center, The Royal Marsden NHS Foundation Trust and The \\ Institute of Cancer Research, 123 Old Brompton Road, London SW7 3RP, UK \\ *Author for correspondence: Tel.: +44 0292068 8562; HopkinsonJB@cardiff.ac.uk
}

\begin{abstract}
Aim: To investigate self-management of dietary intake by colorectal cancer patients receiving chemotherapy. Methods: A questionnaire was administered to 92 patients with nonmetastatic colorectal cancer receiving chemotherapy treatment at a UK cancer center in 2018-19. A maximum variation sample of 20 patients who completed the questionnaire were interviewed. Results: More than three in five patients were at nutritional risk but fewer than one in five were concerned about dietary intake or weight. Selfmanagement of diet and weight was inconsistent with achieving the nutritional intake recommended by clinical guidelines on nutrition in cancer. Conclusion: There is potential for psychoeducation to support change in self-management of nutritional risk, with implications for better treatment tolerance and outcomes including quality of life.
\end{abstract}

First draft submitted: 27 December 2019; Accepted for publication: 8 February 2020; Published online: 7 August 2020

Keywords: chemotherapy $\bullet$ colorectal cancer $\bullet$ diet $\bullet$ mixed-methods $\bullet$ nutrition $\bullet$ psychosocial $\bullet$ qualitative $\bullet$ research • self-management $\bullet$ weight

This research is about self-management of diet and weight by patients with nonmetastatic colorectal cancer during chemotherapy treatment.

\section{Background}

Eating problems during cancer treatment are common and contribute to malnutrition. Weight loss, an indicator of malnutrition, has been observed in $40-92 \%$ of patients 65 years and older during chemotherapy [1]. Expert consensus is that all patients should have optimal nutritional care throughout the course of their cancer [2]. The aim is to prevent malnutrition, thus improving treatment tolerance, survival and quality of life [3]. Support for self-management of dietary intake and weight is a possible way to help patients maintain their nutritional status and therefore potentiate cancer treatment.

\section{Research about eating \& gastrointestinal cancer treatments}

In 2011, Baldwin et al. [4] conducted a systematic review of oral nutritional interventions in malnourished cancer patients. The analysis of 13 studies with 1414 patients, found improved nutritional intake and quality of life. The meta-analysis was of mixed cancer sites and stages, therefore did not reveal if sub-groups of patients are more likely to benefit from oral nutritional support. In the same year, 2011, Elia critically reviewed studies of oral nutritional support during treatment in patients with gastrointestinal cancer [5]. He concluded that the effect on survival and other outcomes is unknown because studies were of mixed cancer stages, compliance with oral supplements poor and groups of well nourished (who perhaps cannot benefit greatly from nutritional support) mixed with 
malnourished patients with sample sizes insufficient to conduct robust subgroup analysis. He draws attention to the proven difficulty in improving dietary intake in real-life situations, for example, when a patient becomes too unwell during treatment to adhere to recommended interventions However, it is known that weight loss before or during cancer treatment can negatively influence treatment outcomes, even in people who are overweight [2].

Our own systematic search of the literature published since 2011, demonstrated that some subgroups of cancer patients are more likely than others to benefit from nutritional support during cancer treatment. Patients with head and neck and gastrointestinal cancers are particularly likely to benefit [6].

In patients with gastrointestinal cancer, preoperative oral nutritional supplements can reduce postoperative surgical site infection rate, postoperative weight loss [7] and serious complications [8]. Compliance with oral nutritional supplements in these patients is variable and independent of weight loss, appetite and comorbidity, but is dependent on information and education [9]. In patients with colorectal cancer followed up after radiotherapy for a median 6.5 years, those who received individualized nutritional counseling to maintain protein and energy intake experienced less treatment toxicity, better quality of life and lower mortality rate compared with patients randomized to either oral supplements and usual diet or to usual diet $[6,10]$. It is not known if nutritional care can achieve similar results for patients receiving chemotherapy for colorectal cancer.

Aim

To investigate self-management of eating problems in patients with colorectal cancer receiving chemotherapy to explore the potential for reducing the risk of malnutrition, which contributes to poor clinical and patient reported outcomes from treatment.

\section{Question}

What practical, social and emotional factors affect eating during chemotherapy treatment and how are they self-managed by patients?

\section{Methods}

The research was a mixed-methods qualitative study of sequential design to identify factors affecting diet and weight during treatment and how they were self-managed. It was conducted at a cancer center in the UK serving a population of approximately 1.5 million people in $2018 / 19$. The objectives were to refine an existing survey questionnaire to investigate the experience of eating and weight for patients receiving chemotherapy treatment for colorectal cancer. To administer the survey to all eligible patients attending clinics over a 30 -week period $(\sim 100$ patients) and to conduct semi-structured exploratory telephone interviews with a sub-set of survey participants (maximum 30 patients) (Figure 1).

Eligibility criteria were:

- Adults (18 years or older);

- Colorectal cancer diagnosis, nonmetastatic (stage I-III);

- Capacity to consent;

- Receiving chemotherapy.

\section{Development of the survey questionnaire}

We developed a bespoke questionnaire, selecting items from existing questionnaires, designed and evaluated for the identification of eating and weight problems in people with advanced cancer or mixed cancer sites. We utilized the knowledge from our previous systematic review of what is known about eating during cancer treatment [5] and development of a cachexia quality of life measure: the EORTC CAX24 [11]. We then added the Scored Patient Generated Subjective Global Assessment (PG-SGA), which includes the four patient-generated historical components (weight history, food intake, symptoms and activities and function - also known as the PG-SGA short form) for assessing nutritional risk [12,13]. A score of $0-1$ requires no intervention, 2-3 requires nutritional education by a dietitian, nurse or other clinician with pharmacology for symptoms, 4-8 requires intervention by a dietician with support from nurse and other clinicians and a score $\geq 9$ indicates a critical need for symptom management and nutritional intervention. Questions about socio-demographics were also included. People affected by cancer $(\mathrm{n}=10)$ were invited to pilot the questionnaire using a think-aloud technique during completion, and their feedback informed the final version (see Appendix I). 


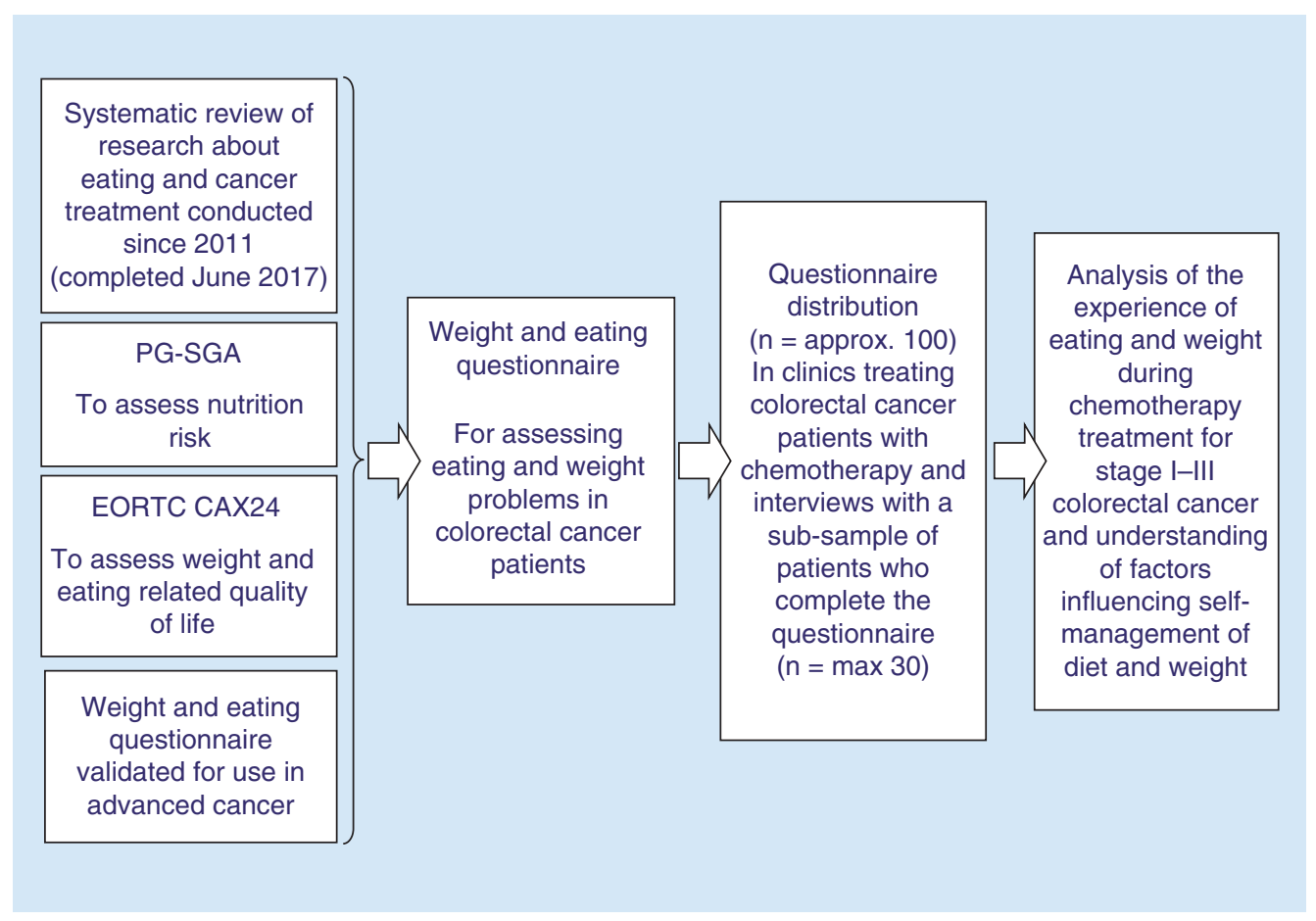

Figure 1. Study design.

EORTC CAX24: The European Organization for Research and Treatment of Cancer quality of life questionnaire cachexia module; PG-SGA: Patient Generated Subjective Global Assessment.

\section{Data collection}

Clinic staff identified eligible patients and introduced them to the researcher (C Kazmi) who offered a study pack comprising of a Study Brochure and the survey questionnaire. The researcher invited the patient to complete the survey during their clinic visit or to return it by post in a stamped addressed envelope. Completion of the questionnaire was taken as evidence of consent to participate [14].

\section{Interviews}

Semi-structured telephone interviews were conducted with a maximum variation sample of patients who complete the questionnaire (C Kazmi). The purpose was to explore the practical, social and emotional experience of eating through treatment and self-management of diet and weight. Interviews were performed until data saturation was achieved, defined as when three consecutive interviews produced no new issues [15].

\section{Analysis}

Survey data were entered into IBM SPSS for windows version 25 (C Kazmi) and analyzed using descriptive statistics and graphical representations of data (JB Hopkinson) to determine the extent and pattern of eating and weight problems.

Interview data were audio-recorded and transcribed verbatim by a professional transcription company. Transcriptions were coded in NVivo computer software (C Kazmi), to assist with data management. Transcripts and coding were read and re-read, then categorized inductively by two researchers working independently (C Kazmi and JB Hopkinson). The researchers compared their analysis and agreed final categorization of the data. Categorization and the identification of relationships between categories conformed to Miles and Huberman's 'mixed strategy' for case-study analysis [16]. Themes relating to the categories were identified (C Kazmi and JB Hopkinson) and then organized using an approach informed by hermeneutic phenomenology [17] to provide the description of physical, social and emotional experience and management of weight and eating during chemotherapy treatment, which revealed possible explanations of the survey results (JB Hopkinson). 
Wales NHS Ethics Committee 3 gave ethical approval for the study. Findings were shared with participants with consent. Anonymity was protected during reporting with patients denoted by P followed by their participant number.

\section{Results}

The survey questionnaire was returned by 52/92 patients (response rate 57\%) with nonmetastatic colorectal cancer, who were receiving treatment from November 2018 to June 2019. In this time period, 132 eligible patients attended 70 clinics. Those not approached were, in clinic at a time the researcher was with another patient or not in attendance $(\mathrm{n}=27)$, did not speak English $(\mathrm{n}=2)$, were judged by a clinician as too unwell to approach $(\mathrm{n}=7)$, or declined to take part $(n=4)$. Telephone interviews were then conducted with a maximum variation sample of 20 patients who completed the questionnaire to explore factors influencing nutritional self-care (Table 1).

\section{Eating problems \& nutritional impact symptoms}

Participants were asked if they were eating less or more than before the start of their treatment. $26 / 50(52 \%)$ of the patients who provided a response were found to eat less and 12/51 (24\%) found to eat more. When asked about the nature of any eating difficulty, more than half reported lack of interest in food, feeling full too quickly and feeling put-off eating by the amount of food on a plate, with nearly a third forcing themselves to eat and a fifth unable to eat although they wanted to (Table 2).

Participants were also invited to report nutritional impact symptoms. Overall, few symptoms were experienced and when they were, few patients reported severity to be 'quite a bit' or 'very much' (Table 3). Nausea, dry mouth, lack of appetite, diarrhea and frequency of bowel movements were the most problematic. When asked if bowel movements had changed what was eaten, 42/50 (84\%) reported 'little' or 'not at all' and when asked if bowel movements had resulted in eating less, 48/50 (96\%) reported 'little' or 'not at all'.

\section{Changes in weight}

51 patients reported their weight at the time of questionnaire completion. The $32 \mathrm{men}$ in the sample had a mean weight of $86 \mathrm{~kg}$ (median $82 \mathrm{~kg}$, range 67-121 kg) and the 20 women had a mean weight $78 \mathrm{~kg}$ (median $75.5 \mathrm{~kg}$, range 51-22 kg). Patients were also asked to report their weight 6 months prior to completing the questionnaire. A total of 48 patients reported their weight at the two time points. Weight gain was reported by $12 / 48$ (25\%), $32 / 48(67 \%)$ reported weight loss and 4/48 (8\%) were weight stable. Of the 19/48 (40\%) patients who had lost more than $5 \%$ body weight, $11 / 19(58 \%)$ had lost weight during their chemotherapy treatment.

\section{Scored PG-SGA (nutritional risk screening)}

A total of 31 men completed the scored PG-SGA, with a mean score of 4.2 (median 3.0, range 0-17) and 20 women completed the measure, with a mean score of 4.0 (median 3.0, range $0-11$ ). In total, 33/51 (67\%) were at 'nutritional risk' with a PG-SGA score of $\geq 2.11$ men (35.5\%) and seven women (35\%) were in the category 'requiring no intervention'. Half of both the men 15/31 (48\%) and women 10/20 (50\%) were at nutritional risk with score $2-8$, where nutritional care and symptom management by the oncology team are important, while five men (16\%) and three women (15\%) were in the category indicating 'critical need for intervention' (see Figure 2).

\section{Emotional impact of changes in eating \& weight}

Despite more than two-thirds of participants being at nutritional risk, most were unconcerned about their eating or weight (Figure 3). When asked if they were concerned about eating, a high proportion, 40/51 (78\%) responded no and similarly, when asked if they were concerned about their weight, 40/50 (80\%) responded no.

Participants also rated the intensity of possible concerns. When asked if concerned about appetite, 51/52 (98\%) reported 'not at all' or 'a little', and when asked to rate worry about eating, 49/51 (96\%) reported 'not at all' or 'a little'. Of the 33 participants who were at nutritional risk with a PG-SGA score 2 or greater, 33/33 (100\%) reported 'not at all' or 'a little' concern about appetite, and 31/32 (97\%) reported 'not at all' or 'a little' worry about eating.

\section{Nutritional information \& advice}

A total of 19/51 (36.5\%) participants had received information about diet and nutrition from a healthcare professional and more than half, 28/51 (54\%), had gathered dietary information from other places (see Table 4.). 


\section{Table 1. Sample characteristics.}

\begin{tabular}{|c|c|c|}
\hline Variables & Survey sample & Interview sample \\
\hline \multicolumn{3}{|l|}{ Gender (n \%) } \\
\hline - Females & $20(38.5)$ & $>9(45)$ \\
\hline - Males & $32(61.5)$ & $11(55)$ \\
\hline \multicolumn{3}{|l|}{ Age (years) } \\
\hline - Mean & 67 & 64 \\
\hline - Median & 68.5 & 62 \\
\hline - Range & 36 to 86 & 48 to 84 \\
\hline \multicolumn{3}{|l|}{ Ethnicity (n \%) } \\
\hline - White British & $51 / 52(98)$ & $19 / 20(95)$ \\
\hline \multicolumn{3}{|l|}{ Education (n \%) } \\
\hline - No formal qualification & $10(19)$ & $0(0)$ \\
\hline - Trade qualification & $8(15)$ & $3(15)$ \\
\hline - Higher degree & $10(19)$ & $6(30)$ \\
\hline \multicolumn{3}{|l|}{ Living with (n \%) } \\
\hline - Partner/spouse & $38(73)$ & $16(80)$ \\
\hline - Family & $4(8)$ & 0 \\
\hline - Alone & $10(19)$ & $4(20)$ \\
\hline \multicolumn{3}{|l|}{ Cancer treatment ( $\mathrm{n} \%$ ) } \\
\hline - Surgery & $48(92)$ & $20(100)$ \\
\hline - Stoma & $19(38)$ & $8(44)$ \\
\hline - Chemotherapy & $52(100)$ & $20(100)$ \\
\hline \multicolumn{3}{|l|}{ Time on treatment in weeks } \\
\hline - (median, mean, range) & $8.5,11.6(1-49)$ & $6.0,11.2(4-28)$ \\
\hline \multicolumn{3}{|l|}{ Comorbidity (n \%) } \\
\hline - None & $19(36.5)$ & $8(40)$ \\
\hline - Diabetes & $6(11.5)$ & $3(15)$ \\
\hline - Heart disease & $6(11.5)$ & $3(15)$ \\
\hline - Multiple & $6(11.5)$ & $2(10)$ \\
\hline PG-SGA (median, mean, (range) & $3.00,4.12(0-17)$ & $3.00,4.1(0-11)$ \\
\hline - Nutritional risk score $\geq 2$ (n \%) & $33 / 51(67)$ & $13 / 20(65)$ \\
\hline \multicolumn{3}{|c|}{ Visual analog scale (median, mean, range) } \\
\hline - Eating-related concern & $0.5,1.8(0-9.5)$ & $0.95,2.31(0-7.5)$ \\
\hline - Eating-related distress & $0.45,1.1(0-9.5)$ & $0.50,1.41(0-7.5)$ \\
\hline - Weight-related concern & $1.2,2.3(0-10)$ & $1.45,2.44(0-7.5)$ \\
\hline - Weight-related distress & $0.5,1.5(0-9.5)$ & $0.50,1.41(0-7.5)$ \\
\hline Eating change ( $\mathrm{n} \%$ ) & $36 / 51(71)$ & $12 / 20(75)$ \\
\hline - Eating more & $12 / 51(23.5)$ & $6 / 19(32)$ \\
\hline - Eating less & $26 / 50(52)$ & $8 / 18(44)$ \\
\hline Weight in $\mathrm{kg}$ (median, mean, range) & $78.0,100.44,(50.8-121.6)$ & $82.9,84.94(57.2-121.6)$ \\
\hline \multicolumn{3}{|c|}{ Weight change over 6 months based on self-report of actual weight ( $\mathrm{n} \%$ ) } \\
\hline - Stable & $4 / 48(8)$ & $3(15)$ \\
\hline - Weight loss $<5 \%$ & $13 / 48(27)$ & $7(35)$ \\
\hline - Weight loss $>5 \%$ & $19 / 48(40)$ & $3(15)$ \\
\hline - Weight gain & $12 / 48(25)$ & 7 (35) \\
\hline \multicolumn{3}{|c|}{ Weight change since commencing SACT based on self-report of YES/NO ( $\mathrm{n} \%$ ) } \\
\hline - Weight has changed & $36 / 52(70)$ & $12 / 20(60)$ \\
\hline - Weigh less & $18 / 50(35)$ & $5 / 18(28)$ \\
\hline - Weigh more & $18 / 50(35)$ & $6 / 18(33)$ \\
\hline
\end{tabular}

However, there was no correlation between receiving or seeking nutritional information and PG-SGA score. A majority $41 / 50(82 \%)$ responded 'not at all' to a question asking if more should be done to help them eat.

\section{Interview findings}

\section{Sample}

The interview sample $(n=20)$ was selected to be representative of the whole sample $(n=52)$. Similar proportions reported weight change, weight loss or weight gain during their chemotherapy treatment, although a lower proportion had experienced $>5 \%$ weight loss over 6 months (Table 1 ).

A range of practical, social and emotional factors influenced eating and the way in which people responded to change in eating habits and weight. 
Table 2. Experience of eating problems.

Problem A little, quite a bit, very much $\mathrm{n}, \mathrm{n}, \mathrm{n}=\mathrm{n} / \mathrm{N}(\%)$

Put off by too much on plate

Feeling full too quickly

Lack of interest

Trouble eating

Problems with liquids

Problems with solids

Forced self to eat

Put off by smell

Food texture unpleasant

Want to eat but unable

\begin{tabular}{ll}
$21,5,1=27 / 50(54)$ & $23 / 50(46)$ \\
$19,3,4=26 / 50(52)$ & $24 / 50(48)$ \\
\hline $16,8,2=26 / 50(52)$ & $24 / 50(48)$ \\
\hline $15,4,1=20 / 49(41)$ & $29 / 49(56)$ \\
$8,6,2=16 / 51(32)$ & $35 / 51(69)$ \\
\hline $14,1,1=16 / 50(32)$ & $34 / 50(68)$ \\
\hline $11,2,2=15 / 50(30)$ & $35 / 50(70)$ \\
\hline $8,4,2=14 / 49(29)$ & $35 / 49(71)$ \\
\hline $5,4,2=11 / 49(23)$ & $38 / 49(78)$ \\
$8,1,1=10 / 50(20)$ & $40 / 50(80)$
\end{tabular}

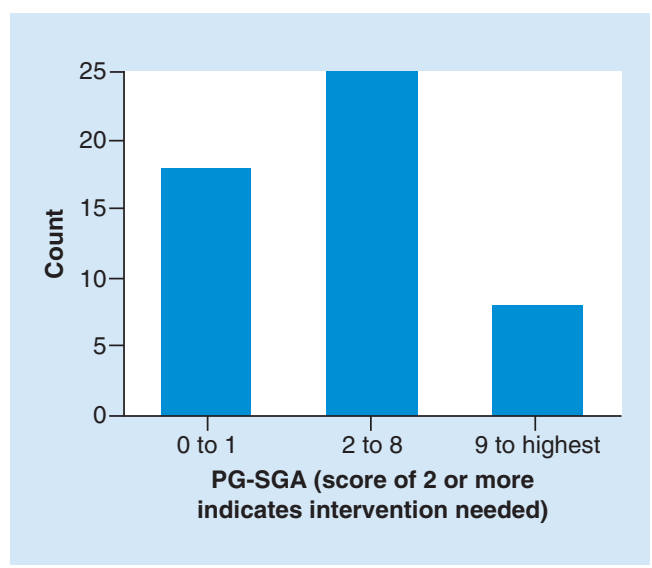

Figure 2. Nutritional risk.

PG-SGA: Patient Generated Subjective Global Assessment.

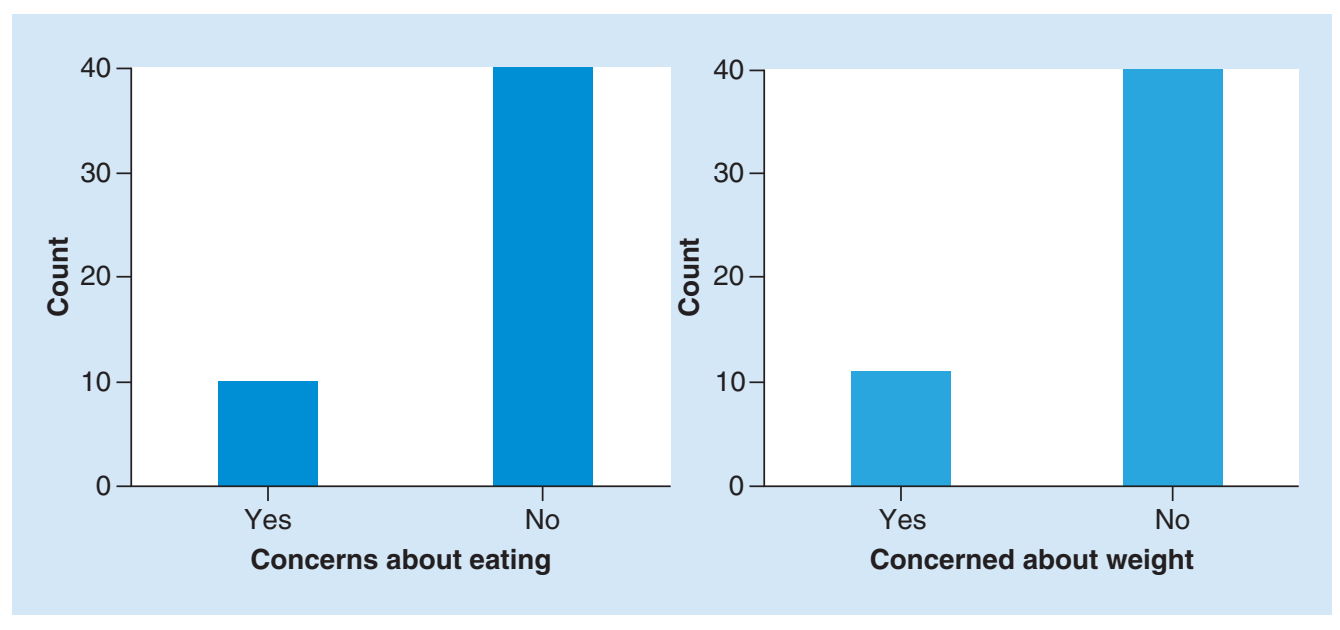

Figure 3. Concern about eating and weight.

Practical factors affecting dietary intake

Common side effects

The participants spoke about chemotherapy side effects and disease symptoms affecting what they were able to eat and drink, consistent with the questionnaire responses; 


\section{Table 3. Nutritional impact symptoms.}

Most problematic nutritional impact symptoms

( $>10 \%$ experienced them 'quite a lot' or 'very much')

Least problematic nutritional impact symptoms

Nausea

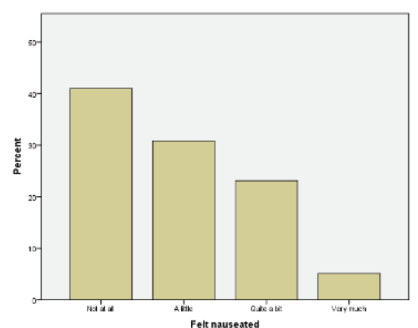

( $<10 \%$ experienced them 'quite a lot' or 'very much')

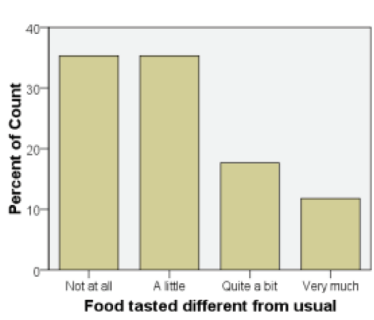

Taste change

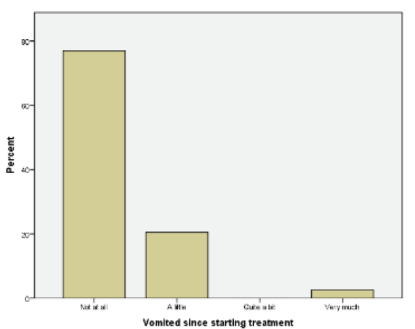

Vomiting

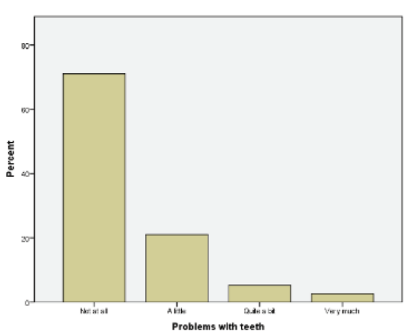

Teeth

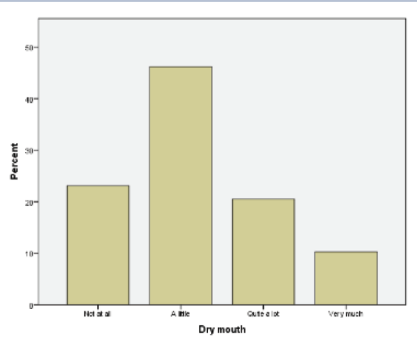

Dry mouth

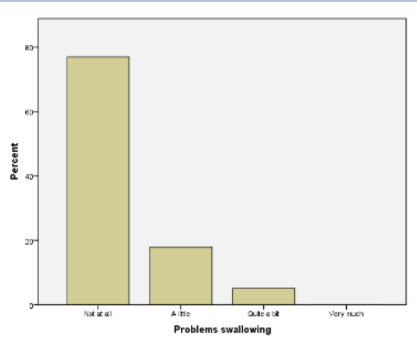

Swallowing

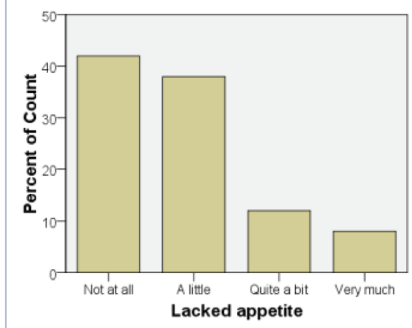

Lack of appetite

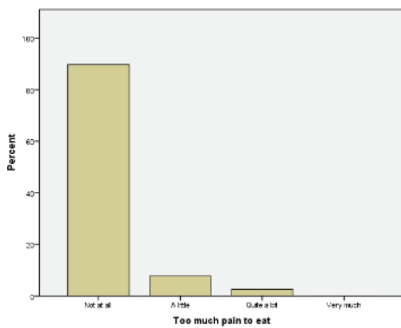

Pain

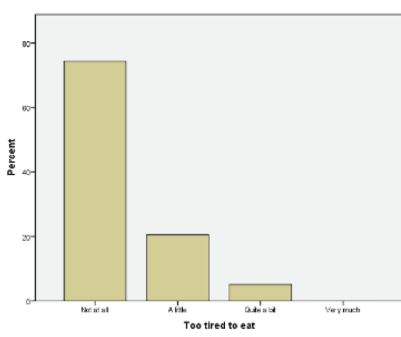

Tiredness

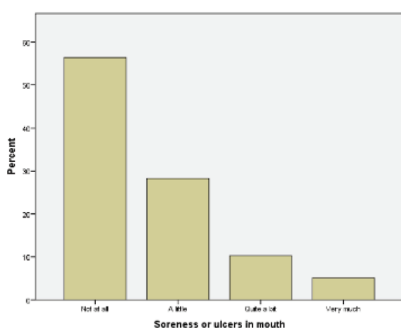

Mouth ulcers 


\section{Table 3. Nutritional impact symptoms (cont.).}

Most problematic nutritional impact symptoms ( $>10 \%$ experienced them 'quite a lot' or 'very much')

Bowel symptoms

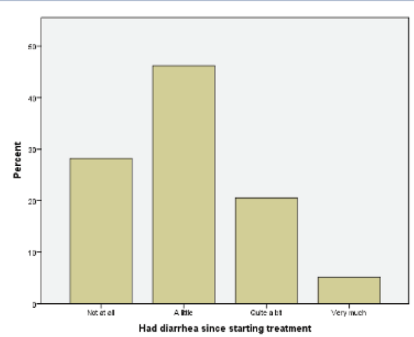

\section{Diarrhoea}

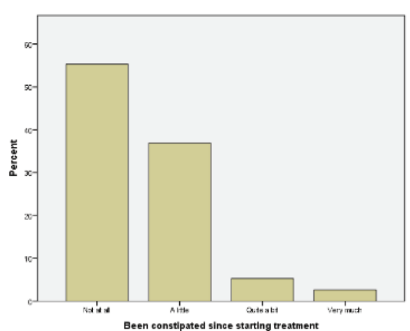

Constipation

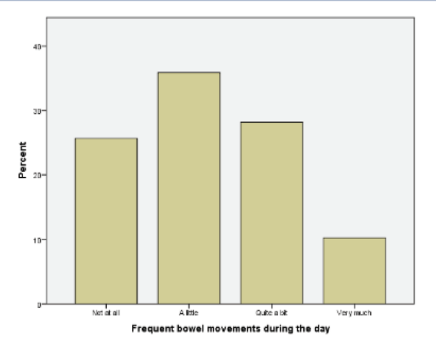

Frequent bowel movements during the

day

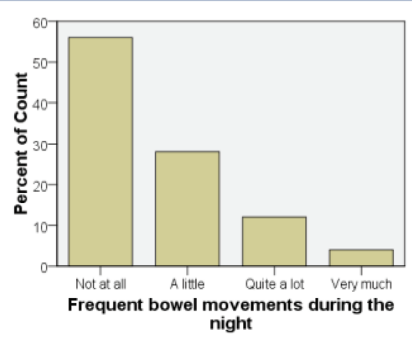

Frequent bowel movements at night

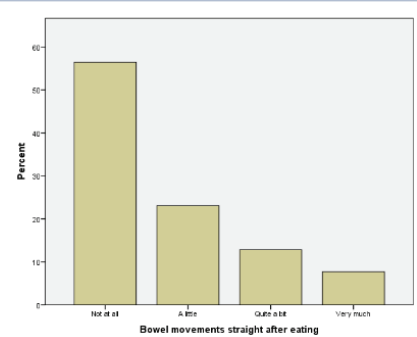

Bowel movements after eating

\section{Table 4. Sources of nutritional information and advice.}

Information from a healthcare Information from other places professional

PG-SGA score between 2 and 8 PG-SGA score $\geq 9$ offered offered healthcare professional healthcare professional advice advice

\begin{tabular}{|c|c|c|c|c|}
\hline Men & $12 / 32(37.5 \%)$ & $17 / 31(53 \%)$ & $6 / 15(40 \%)$ & $3 / 5(60 \%)$ \\
\hline Women & $7 / 19(35 \%)$ & $11 / 19(55 \%)$ & $4 / 10(40 \%)$ & $1 / 3(33 \%)$ \\
\hline Total & $19 / 51(36.5 \%)$ & $28 / 51(28 \%)$ & $10 / 25(40 \%)$ & $4 / 8(50 \%)$ \\
\hline
\end{tabular}

PG-SGA: Patient Generated Subjective Global Assessment.

"Even water tasted funny, I had a job to drink water, um so food just tasted absolutely dreadful. .. the first couple of days, I'd have a bit of constipation and then diarrhoea set in and it would be on and off for the rest of the cycle." (P31)

However, side effects were fewer in number and less severe than expected: 
"I get no sickness. Nothing. I think I'm one of the lucky ones."

When asked about what they had eaten the day before the interview, more than half described their intake as 'normal' or similar to before their cancer diagnosis.

\section{Strategies to eat \& drink}

An approach for managing chemotherapy side effects at home used by some was to follow rules. These might be personal rules but informed by information and advice given by oncology staff. Advice to eat before taking chemotherapy medication was an important influence on the pattern of eating or the decision to eat at all;

"My portion sizes are smaller, um, because of my medication, I have to eat before I have my medication. So, I have an alarm on my phone, to flash lunchtime, then I go oh right okay, I've gotta remember to eat something." (P33)

Public health messages disseminated to the general population about what to eat and drink for health and well-being also influenced personal rules;

"We've always eaten healthily. Er, there's no frying pan in this house. We don't have sugar. We don't use sugar. I had a weak coffee, but we usually drink boiled water. Both of us, yea, it's all fresh vegetables or, frozen vegetables.... Weve always eaten healthy, healthy salads and fresh vegetables, fruit. We love our fruit, so it's not much different to what it was before the cancer set in." (P29)

A temporary thing

The side effects described as most hindering to eating were nausea and sickness, diarrhea, loss of hunger/appetite, cold sensitivity to fluids and food leading to difficulty in keeping hydrated and also in handling food and drink. Any disruption to eating caused by chemotherapy side effects was 'just one of these things you've got to put up with' (P12) and expected to be temporary;

"It's all part of the chemo, once chemo finishes, I think I will be a lot better."(P38)

\section{Social factors affecting dietary intake}

Disruption of day-to-day activity

The change in day-to-day activity, particularly for those who had stopped working during treatment, was a factor influencing dietary intake. P5 was concerned about weight gain;

"Once I go back to work my diet is gonna change, because, obviously at home you do tend to pick and I don't think that's doing me any good. It's just, probably, boredom and stuff like that, I mean if I go out and come back in, I'll have a biscuit with a cup of tea, or a piece of cake and a cup of tea. It's one of those things that I wouldn't do in work." (P5)

Physical activity or exercise were talked about along with dietary intake, as important for managing weight. Some participants spoke about physical activity or exercise to have been an important part of their lives. Concern was expressed because of the difficulty in remaining physically active during treatment and the implications, especially for those who did not want to gain weight;

"I should be doing a bit more exercise, but I can't do it at the moment, I tried to walk a little bit, but it's very, very tiring and I'm not very steady on my feet." (P20)

A particular challenge for patients with a stoma who wanted to remain active, was concern about going out, because of the risk of a leak from the stoma bag. This affected their activity and also what they ate, with restriction of food and fluid intake being a way to manage the risk;

"I've got a colostomy bag... . That's kind of encouraged me to not eat very much.....If I'm going out in public,

I won't eat... If I'm going out, I'll avoid eating while I'm out, and then I'll eat when I get back home." (P17)

Having another person either to feed, or who offered help with food preparation was helpful for those experiencing problems eating. To care for others, or care shown by others in providing food, was experienced as more than an offer of food for the body, but fuel for a sense of well-being. Patients who experienced the most difficulty finding the energy and motivation to attend to their nutritional intake were those who lived alone; 
"Because it's only me in the house... I try and cook, I cook dinner, that's only once a week now, it used to be every day." (P38)

A self-management routine

When disruption in day-to-day activity was recognized as a risk to either taking adequate nutrition or eating too much, a proactive approach could include establishing a routine. The routine might be to engage in a distracting activity to avoid overeating. For example, 'doing jigsaws' (P33).

It might also include adapting an approach successful in managing a precancer problem;

"What I always find helps with my weight, to go to class every week, to weigh myself... I used to enjoy going to the Slimming World classes to get tips from other people and the support 'oh, well done' from the other people. My husband's overweight, he's gone to (the chemist) the last three weeks now to weight there, which only costs us seventy pence, weigh and a little slip comes out, and I started that now last week. . So, we are supporting each other that way." (P33)

P33 has not only resuming an old activity that has previously enabled her to manage her weight, but also engaged her husband in her weight management plan. A mutually beneficial activity is established, as a helpful routine within their personal capabilities and financial resources.

Return to former activities, dietary habits and management of health was a recurring theme. The goal being to get back to normal;

"I just think keep going and trying to get back to normal and not sit around too much, you know, and get back to your normal activities." (P27)

Normal food and eating habits provided some reassurance of recovery;

$$
\text { ".... it was the best I was feeling for, for ages... ., I was able to eat normally" (P28) }
$$

Emotional factors affecting dietary intake Getting better

Many patients were seemingly unconcerned about their diet, nutritional intake or eating habits. They gave accounts of improvement in what they were able to eat and drink, which assured them all was well, that additional information and advice on food, eating and nutrition was unnecessary and there was nothing more to be done;

"I'm sure I read somewhere, on one of the leaflets that you could ask for dietary advice. And. . but I didn't really feel I needed it." (P8)

Factors described as evidence of being on a pathway of recovery were 'mind matters,' 'healthy eating,' and 'benefits of weight loss.'

Mind matters

Recurring were comments about positive thinking and having the right attitude for recovery;

"Although I've reduced my food, you know, for the bag and things, I don't let it get to me... I just think, oh well. . I think a positive mind-set is the main thing. . . faith that everything is going to be okay." (P17)

Clinical staff were seen responsible for medical treatments to manage the disease and its symptoms. Personal responsibility was to have a positive attitude.

Healthy eating

Having the right attitude included eating the right foods for recovery and health. A small number of patients 'worried about the nutritional side of things' (P31). For these people, the right foods were believed to be the balanced diet recommended for the healthy population, to include fruit and vegetables every day. This proved challenging;

"I was concerned because, normally, my intake in nutrients and vitamins is normally quite high. I felt that because I was drawn to mainly just having lots of sandwiches and what I wanted, I was sort of fighting it at first, because I thought no I need to have my usual fruit and veg. . in the end, I just thought, right, I just need to give in to how I'm feeling, because there is enough going on without me fighting myself over diet." (P7) 


\section{Benefits of weight loss}

It was more often that the patients considered weight rather than nutrition. They spoke about their current weight and weight change evidencing improvement in health;

"I'm keeping to $11 \frac{1}{2}$ stone now. I'm happy with that. I needed to lose weight anyway, I was up to $14 \frac{1}{2}$ stone, that's no good, that's no good to anybody, but it's off now and I'm very pleased about it." (P29)

Most gave an account of their experience of surgery. Typically, this was of rapid weight loss followed by weight gain during the post-operative recovery period, which might be spontaneous or the result or effort of consciously 'building up again' (P18). The benefits of weight loss were emphasized, particularly by those who had been overweight before the surgery, but also by patients who were not.

"I had the operation... that just knocks back your appetite, erm, whether there's some other more medical reason why I lost, I don't know, but the result was I lost 8 or 9 kilos. It wasn't a problem because, although I not overweight at all, it possibly didn't do me any harm to lose that." (P23)

Family members reinforced beliefs about the benefits of weight loss;

“.. my family are very pro me losing weight. I have that pressure." (P20)

Patients who were gaining weight but who did not want to return to or become overweight, spoke about weight being out of their control during treatment. This might be because of the advice given to eat before taking oral chemotherapy to minimize treatment side effects;

"I'd rather not put on any more weight but ... I guess I'm between a rock and a hard place really. I mean, I do need to eat so as I can take my chemo drugs." (P16)

Or, it was explained that steroid treatment 'does increase your appetite; (P25) and change in everyday activity, for example 'not being in work' (P25) had impact on dietary intake and weight.

\section{Nothing to be done \& taking control}

Although there was a dominant shared narrative of weight and weight gain being out of control during cancer treatment - partly because of the disease treatment and treatment side effects - there was a competing narrative. This was the dominant narrative throughout six interviews with patients across the spectrum of nutritional risk, assessed using the PG-SGA, three of whom were weight gaining and three weight losing. It was of overtly taking control to aid recovery, in spite of this being difficult;

"I make myself eat, even when I don't want to. I try and keep it to three or four reasonable health meals a day,, cos, as far as I can concentrate on good quality food as well than just getting something into me, and I do as much exercise as I can tolerate. ... It's you know, taking control and sometimes not feeling like it, but knowing that it, in the long run it's what my body needs and how I'm gonna recover. . . A bit of control over what's going on with me, I suppose, it's keeping myself in the best condition that I can while I can't exercise and eat what I would normally, I want to try and maintain at least a part of it. It gives you focus." (P18)

The two narratives of being 'out of control' versus taking control, may be intertwined within a patient's story. This was evident in accounts of internet use. P23 is an example of someone who talks about the internet as a source of information for managing diet and weight but who has confidence in his existing knowledge of diet and nutrition, so considers there is nothing to be done;

"One is able to Google. . . what's good to eat, which is probably not very clever. It's best to ask people, but err, no try and have a reasonable diet, you know, the reasonable fruit and vegetables." (P23)

Five patients actively avoided searching the internet for information about diet and weight 'you get into a lot of misinformation' (P9). It was considered a worrying or frightening thing to do. Thus, to do nothing was a passive way of taking control;

"I specifically avoided that, um, I know if you look something up on the internet, generally you just worry yourself to death, so I made a particular point of not looking stuff up." (P25) 
Talking about the internet revealed the intertwined narratives of actively taking control by managing nutritional intake and physical activity through rules, routine and self-help, as compared with a more passive approach of drawing on existing knowledge and experience to find one's own way and wait for treatment-related problems to pass. For those taking control passively, the intention was always to 'just do things normally really' (P17) or return to normal;

\section{"I was never really a big eater; my normal standard weight is seventy kilos (....) I'm trying to get my weight back to normal." (P25)}

\section{Making sense of the survey results \& interview findings}

The majority of patients were at nutritional risk, but unconcerned about diet, eating and weight. An explanation for this lack of concern is the sense of control gained from taking the same diet as prior to their cancer diagnosis or from a belief in being on a pathway back to normal. This belief is reinforced by the improvement in dietary intake and recovery of weight loss post-surgery and prior to commencing chemotherapy, accompanied by fewer than expected side effects of chemotherapy. The assumed return to eating normally equated with recovery, contributed to ambivalence about actively managing dietary intake and the decision that there was nothing to be done until after chemotherapy treatment. A minority of patients gained a sense of control and well-being during chemotherapy treatment by actively seeking information and self-managing nutritional intake and weight. This minority spanned all categories of nutritional risk and included those who were both weight gaining and weight losing. Education to disrupt the goal of eating as normal (pre-cancer) may be a way to facilitate change in self-management. There is potential for disruption of the belief that eating as normal is good, to enable a greater number of patients to actively self-manage, whether they are weight losing, weight gaining or at any level of nutritional risk.

\section{Discussion}

In this study, a majority of patients with nonmetastatic colorectal cancer reported changes in eating habits during chemotherapy treatment and two-thirds were assessed to be at nutritional risk. Malnutrition can lead to weight loss or weight gain. 25 percent had gained weight and $67 \%$ had lost weight over a six-month period (37\% after starting chemotherapy treatment). Concern regarding eating was reported by one in four $(25 \%)$ who had gained weight and one in five (19\%) who had lost weight, over 6 months. Thus, there was a large group of patients who had no eating concerns despite being at nutritional risk.

The interviews revealed a dominant narrative of confidence in self-management of eating and weight, reinforced by weight gain accompanying recovery following surgery, which led to a belief that further dietary information or advice was unnecessary. Experience of overcoming eating problems and regaining weight lost pre- and immediately post operatively, plays an important role in how people go on to manage eating while on chemotherapy. Confidence that recovery was in progress was further reinforced by the experience of fewer and less intense nutritional impact symptoms than were expected while on chemotherapy. Also, important to note, is that some patients welcomed weight loss. Of these, some welcomed weight loss because they had been overweight, as has previously been reported [18]. However, others did so despite having been underweight.

\section{Interest in nutritional care}

An online survey of 96 patients, most living in south or south-east England, one-third with advanced disease, and one-third with breast cancer, reported dissatisfaction with nutritional care and wanted additional advice on diet and nutrition during treatment to help them manage side effects of chemotherapy, weight changes and what they eat [19]. Interview based studies evidence that patients find eating problems distressing and lack appropriate dietary instructions [20,21]. Yet there is competing evidence. Patients have also been found reluctant to engage in nutritional care. A study conducted in Turkey of mixed cancer sites reported that more than a third of patients declined participation because of fatigue or disinterest in nutritional support [22]. Our study demonstrated that diet and nutrition was of concern to only a minority of patients, despite the fact that it should have been of concern to all at nutritional risk. A majority were content with the nutritional advice and information available to them. The sample demographic was typical in terms of age, gender, ethnicity and domestic status of patients receiving treatment for colorectal cancer when compared with 857 colorectal cancer patients from 29 centers across the UK [23]. Lack of concern surrounding dietary intake and nutrition during chemotherapy for colorectal cancer may manifest beyond the study center location in South Wales, UK. 


\section{Clinical guidelines}

Nutritional counseling is the most often used intervention for patients with cancer who have a functioning gastrointestinal tract and are malnourished [24]. It can improve nutritional status in patients receiving chemotherapy [25]. In China, patients with gastric cancer receiving chemotherapy were randomized to intensive nutritional counseling or usual nutritional care $(\mathrm{n}=144)$. The intervention group had improved calorie and iron intake throughout treatment and fewer treatment interruptions compared with the usual care control [26]. Critical components of nutritional counseling are to explain reasons for and agree goals of nutritional recommendations and to motivate the patient to adapt to the nutritional demands of their cancer [2]. These components are consistent with behavioral change theories and strategies known to facilitate food behavior change beyond the context of cancer care [27].

Personalized nutritional counseling and support for physical activity is recommended by the European Society for Clinical Nutrition and Metabolism (ESPEN) Clinical Guideline on nutrition in cancer, to stabilize weight and prevent loss of muscle mass and function [2]. counseling is recommended to support dietary adaptation using regular foods, fortified foods and oral food supplements [24] to achieve an intake of $25-30 \mathrm{kcal} / \mathrm{kg} / \mathrm{day}$ and $1.2-1.5$ $\mathrm{g}$ protein $/ \mathrm{kg} / \mathrm{day}$ (or intake based on an estimate of energy needs) [24]. However, this knowledge alone may not change self-management of eating during treatment. Beliefs surrounding eating, such as perceived health benefits, not just symptoms such as taste change, constrain and enable management of eating problems [28]. Our study reveals participants were unconcerned about eating and mostly unaware of nutritional risk, and nutritional considerations influenced diet of only a minority. This minority were concerned to take control by eating the healthy diet promoted to prevent or reduce risk of disease. Dietary management has similarly been found an important way of feeling in control for breast [29] and prostate [30] cancer patients on treatment.

For a majority of our study participants, food and fluid intake was influenced by a desire to either continue as normal, or the hope of returning to normal. Thinking and behavior was driven by the quest for normal recovery and thus giving comfort. It was feeling not fueling that was important to self-management of diet, eating and weight during treatment. This suggests that, for care to be effective in supporting self-management of nutritional risk during chemotherapy treatment, it should focus not only on fueling the body, advising the best nutrient intake, but also create a feel-good factor.

\section{A personal reference point}

Normal eating habits or usual dietary intake prior to receiving a diagnosis of colorectal cancer was used by a majority of patients, as the reference point for recovery and indicator of health. This has implications for nutritional care during chemotherapy treatment. It seems likely that to be effective, nutritional counseling should advise adaptations as close as possible to the patient's normal intake and eating pattern, as has previously been recommended to support patients receiving radiotherapy [10]. It also raises a question about the potential for psychoeducation to change the reference point. For example, if the advised reference point was stable weight through treatment, as recommended by ESPEN, this may facilitate a guideline compliant dietary intake for patients receiving chemotherapy. In other words, it may encourage dietary intake, which can reduce the risk of interruptions to treatment schedule and improve quality of life through improved nutrition [24]. The approach may be particularly helpful for patients who self-manage eating during chemotherapy treatment by creating personal rules informed by healthcare professional advice.

\section{Limitations}

This research was a single center study in the UK and was about nutritional care of patients receiving a single treatment modality, chemotherapy. Almost all patients with Stage I disease are treated by surgery alone, therefore it is likely that the analysis and conclusions are based on data collected from patients with Stage II-III colorectal cancer. The transferability of the findings to other disease stages, cancer treatments and other geographical locations in the UK and beyond, should be investigated with a larger sample size. This would permit robust statistical tests of the propositions generated by the analysis and stratification to enable comparison of rectal and colon cancers and comparison of weight losing, weight gaining and weight stable patient groups.

Data collection was performed by a social scientist who then conducted the analysis in partnership a nurse academic. The findings have been tested for credibility through discussion with service users, cancer clinicians, clinical academics and researchers practising in England and Wales. Evaluation of credibility should be extended to include multidisciplinary cancer teams across all four nations in the UK and beyond. 


\section{Conclusion \& future perspective}

Patients with nonmetastatic colorectal cancer receiving chemotherapy treatment were at nutritional risk, but few were concerned about dietary intake or weight. A minority sought to take control of their nutritional intake by striving to consume the healthy diet recommended to reduce risk of disease in the healthy population. A majority took comfort from continuing to eat normally or striving to return to normal precancer dietary intake. Neither approach to self-management of eating and weight was consistent with achieving the nutritional intake recommended by the ESPEN Clinical Guideline on nutrition in cancer [24]. There is potential for psychoeducation to support change in self-management of nutritional risk, with the implication of a future with better chemotherapy tolerance and outcomes, including quality of life.

\section{Summary points}

- Malnutrition during cancer treatment is associated with poor treatment tolerance, survival and quality of life.

- Self-management of dietary intake may be an important way to improve treatment tolerance and outcomes.

- Little attention has been given to the facilitation of optimal dietary intake during cancer chemotherapy.

- We found patients with colorectal cancer receiving chemotherapy to be at nutritional risk, but unconcerned about their dietary intake.

- A minority self-managed by taking the healthy diet recommended to reduce risk of disease, while a majority sought to return to their precancer dietary intake.

- Self-management of dietary intake was inconsistent with achieving the recommended nutritional intake for patients receiving chemotherapy.

- There is potential for supportive nutritional care to potentiate chemotherapy in patients with colorectal cancer.

\section{Acknowledgments}

The authors would like to thank the people with cancer who took part in the study and the healthcare professionals who supported recruitment. We would also like to thank LL Gould, B Burbidge, G Knight and C Zollman, who were members of the study advisory group.

\section{Financial \& competing interests disclosure}

This work was funded by Tenovus Cancer Care, iGrant TIG2018-34. This work was supported by the NIHR Cancer and Nutrition Collaboration. The views expressed are those of the author(s) and not necessarily those of the NIHR or the Department of Health and Social Care Funding. The authors have no other relevant affiliations or financial involvement with any organization or entity with a financial interest in or financial conflict with the subject matter or materials discussed in the manuscript apart from those disclosed.

No writing assistance was utilized in the production of this manuscript.

Ethical conduct of the research

Wales NHS Ethics Committee 3 gave ethical approval for the study.

The authors state that they have obtained appropriate institutional review board approval or have followed the principles outlined in the Declaration of Helsinki for all human or animal experimental investigations. In addition, for investigations involving human subjects, informed consent has been obtained from the participants involved.

Informed consent disclosure

The authors state that they have obtained verbal and written informed consent from the patient/patients for the inclusion of their medical and treatment history within this case report.

\section{Open access}

This work is licensed under the Attribution-NonCommercial-NoDerivatives 4.0 Unported License. To view a copy of this license, visit http://creativecommons.org/licenses/by-nc-nd/4.0/

\section{References}

Papers of special note have been highlighted as: • of interest; $\bullet \bullet$ of considerable interest

1. Caillet P, Liuu E, Simon A et al. Association between cachexia, chemotherapy and outcomes in older cancer patients: a systematic review. Clin. Nutr. 36(6), 1473-1482 (2016).

2. Arends J, Baracos V, Bertz $\mathrm{H}$ et al. ESPEN expert group recommendations for action against cancer-related malnutrition. Clin. Nutr. 36(5), 1187-1196 (2017). 
-• A position paper setting out three key steps for the improvement of nutritional care for people with cancer.

3. Aapro M, Arends J, Bozzetti F et al. Early recognition of malnutrition and cachexia in the cancer patient: a position paper of a European School of Oncology Task Force. Ann. Oncol. 25(8), 1492-1499 (2014).

- A position paper making a case for oncologists to attend to nutritional and functional status throughout the course of anti-cancer treatment.

4. Baldwin C, Spiro A, Ahern R, Emery PW. Oral nutritional interventions in malnourished patients with cancer: a systematic review and meta-analysis. J. Natl Cancer Inst. 104, 371-385 (2011).

5. Elia M. Oral nutritional support in patients with cancer of the gastrointestinal tract. J. Hum. Nutr. Diet 24, 417-420 (2011).

6. Hopkinson J. Psychosocial support in cancer cachexia syndrome: the evidence for supported self-management of eating problems during radiotherapy or chemotherapy treatment. Asian-Pacific J. Nurs. 5, 358-368 (2018).

- A scoping review detailing what is known about self-management and support for self-management of nutritional risk during cancer treatment.

7. Burden ST, Gibson DJ, Lal S et al. Pre-operative oral nutritional supplementation with dietary advice versus dietary advice alone in weight-losing patients with colorectal cancer: single-blind randomized controlled trial. J. Cachexia Sarcopenia Muscle 8(3), 437-446 (2017).

8. Kabata P, Jastrzebski T, Kakol M et al. Preoperative nutritional support in cancer patients with no clinical signs of malnutrition prospective randomized controlled trial. Support. Care Cancer 23, 365-370 (2015).

9. Grass F, Bertrand PC, Schafer M et al. Compliance with preoperative oral nutritional supplements in patients at nutritional risk - only a question of will? Eur. J. Clin. Nutr. 69, 525-529 (2015).

10. Ravasco P, Monteiro-Grillo I, Camilo M. Individualized nutrition intervention is of major benefit to colorectal cancer patients: long-term follow-up of a randomized controlled trial of nutritional therapy. Am. J. Clin. Nutr. 96, 1346-1353 (2012).

- Long-term follow-up (median 6.5 years) of patients with colorectal cancer who received early individualized nutritional counseling and education during radiotherapy showing improved nutritional status, quality of life and survival compared with patients who did not receive the intervention.

11. Wheelwright SJ, Hopkinson JB, Darlington A-S et al. Development of the EORTC QLQ-CAX24, a questionnaire for cancer patients with cachexia. J. Pain Symptom Manage. 53(2), 232-242 (2017).

12. Bauer J, Capra S, Ferguson M. Use of the scored Patient-Generated Subjective Global Assessment (PG-SGA) as a nutrition assessment tool in patients with cancer. Eur. J. Clin. Nutr. 56(8), 779-785 (2002).

13. Abbott J, McKavanagh LTD, Watson J, McCarthy AL, Isenring E. Patient-Generated Subjective Global Assessment Short Form (PG-SGA SF) is a vaild screening tool in chemotherapy outpatients. Support. Care Cancer 24(9), 3883-3887 (2016).

14. Ream E, Pedersen VH, Oakley C et al. Informal carers' experiences and needs when supporting patients through chemotherapy: a mixed methods study. Eur. J. Cancer Care 22, 797-806 (2013).

15. Francis JJ, Johnston M, Robertson C et al. What is an adequate sample size? Operationalising data saturation for theory-based interview studies. Psychol Health. 25(10), 1229-1245 (2010).

16. Miles M, Huberman M (Eds). Making good sense: drawing and verifying conclusions. In: Qualitative Data Analysis. Sage, Thousand Oaks (1994).

17. Van Manen M. Researching Lived Experience: Human Science for an Action Sensitive Pedagogy. The Althouse Press, London (1990).

18. Hopkinson J. Psychosocial impact of cancer cachexia. J. Cachexia Sarcopenia Muscle 5, 89-94 (2014).

19. NIHR Southampton Biomedical Research center (2015). Cancer and Nutrition NIHR infrastructure collaboration: improving cancer prevention and care. For patients. For Clinicians. For researchers. Phase I report. National Institute for Health Research. http://cancerandnutrition.nihr.ac.uk/wp-content/uploads/2016/06/Cancer-Nutrition-Full-Report-FINAL_03-06-16.pdf

20. Boltong A, Keast R, Aranda S. Experiences and consequences of altered taste, flavour and food hedonics during chemotherapy treatment. Support Care Cancer 20(11), 2765-2774 (2012).

21. Cooper C, Burden ST, Molassiotis A. An explorative study of the views and experiences of food and weight loss in patients with operable pancreatic cancer perioperatively and following surgical intervention. Support Care Cancer 23, 1025-1033 (2015).

22. Uster A, Ruefenacht $\mathrm{U}$, Ruehlin $\mathrm{M}$ et al. Influence of a nutritional intervention on dietary intake and quality of life in cancer patients: a randomized controlled trial. Nutrition 29, 1342-1349 (2013).

23. Foster C, Haviland J, Winter J et al. Members of the Study Advisory Committee. Pre-Surgery Depression and Confidence to Manage Problems Predict Recovery Trajectories of Health and Wellbeing in the First Two Years following Colorectal Cancer: Results from the CREW Cohort Study. PLoS ONE 11(5), e0155434 (2016).

- Identifies presurgery self-efficacy and depression to be predictors of recovery in 2 years following colorectal cancer surgery.

24. Arends J, Bachmann P, Baracos V et al. ESPEN guidelines on nutrition in cancer patients. Clin. Nutr. 36, 1-48 (2017).

- Guidelines for multi-disciplinary teams in the identification, prevention and treatment of malnutrition in adult cancer patients, based on best available evidence and expert opinion. 
25. Bourdel-Marchasson I, Blanc-Bisson C, Doussau A et al. Nutritional advice in older patients at risk of malnutrition during treatment for chemotherapy: a two-year randomized controlled trial. PLoS ONE 9(9), e108687 (2014).

26. Xie F-L, Wang Y-Q, Peng L-F, Lin F-Y, He Y-L, Jiang Z-Q. Beneficial effect of educational and nutritional intervention on the nutritional status and compliance of gastric cancer patients undergoing chemotherapy: a randomized trial. Nutr. Cancer 69(5), 762-771 (2017).

27. Spahn JM, Reeves RS, Keim KS et al. State of the evidence regarding behavioral change theories and strategies in nutrition counseling to facilitate health and food behavior change. J. Am. Diet Assoc. 110(6), 799-901 (2010).

28. Bernhardson BM, Olson K, Baracos VE, Wismer WV. Reframing eating during chemotherapy in cancer patients with chemosensory alterations. Eur. J. Oncol. Nurs. 16, 483-490 (2012).

29. Pedersen B, Groenkjaer M, Falkmer U, Delmar C. Understanding the essential meaning of measured changes in weight and body composition among women during and after adjuvant treatment for breast cancer. Cancer Nurs. 40(6), 433-444 (2016).

30. Kassioanos AP, Coyle A, Raats MM. Perceived influences on post-diagnostic dietary change among a group of men with prostate cancer. Eur. J. Cancer Care 24, 818-826 (2015). 